()

CORPUS PUBLISHERS

\section{Corpus Journal of Dairy and} Veterinary Science (CJDVS)

Volume 3 Issue 1, 2022

Article Information

Received date : January 14, 2022

Published date: January 21, 2022

*Corresponding author North D Warner, San Francisco, California, USA

Distributed under Creative Commons CC-BY 4.0

\title{
Need for International Workshops to Deliberate Evidence of Benefits and Risks of Raw Millks
}

\author{
North DW*, Coleman ME and Hull RR
}

San Francisco, California, USA

Opinion

Collaborative holistic approaches that incorporate recent evidence from multiple disciplines or sectors, including microbiology, immunology, and dairy and veterinary science, are crucial to enhancing sustainability, resilience, and health of humans and ecosystems around the world. Recent companion studies published in the Open Access journal Applied Microbiology [1-3] describe transdisciplinary analysis of benefits and risks for foodborne and environmental hazards using evidence map approaches for depicting the 'state of the science' and uncertainties related to both infectious and non-communicable diseases. The evidence documented in these studies [1-3] reflect the scientific advances of the recent decade summarized in the review by Oikonomou and colleagues [4] that describes the composition and functionality of mammalian milk microbiota and consortia naturally present in fresh unprocessed (raw) milks from healthy humans and ruminants. No longer is there consensus in the scientific community that mammalian milks are sterile, an outdated belief strongly based on germophobia of the 20th century. The current body of evidence [1-3] challenges 20th-century notions about microbes naturally present in milks of healthy mammals and merits further deliberation of the quality, veracity, and coherence of available evidence for assessing benefits and risks for regulatory decision making and other policy making. No benefit-risk analysis study was identified in our searches for pasteurizing donor breastmilk or cow milk. Further, much of the available evidence for benefits and risks of raw cow milk [3] is inconsistent with many outdated and unvalidated assumptions that formed the basis for simulations of potential risks to raw milk consumers in the past. One such simulation study by Food Standards Australia New Zealand (FSANZ) in 2009 [5] appeared to us to be driven by selected assumptions that seem to support preconceived pro-pasteurization biases articulated in the 2009 report, rather than to rely on sound scientific data. Many FSANZ assumptions (particularly: extrapolation of pathogen presence and levels in milk from cow feces; minimal effectiveness of temperature control, hygienic practices, Hazard Analysis and Critical Control Points (HACCP) programs, and test-and-hold programs; and high inherent risk) are falsified by the current body of evidence documented in this peerreviewed analysis [3]. From our perspective, recent data from clinical, mechanistic, and outbreak studies do not support the outdated assumptions that raw milk is inherently dangerous and that current hygienic management programs, including HACCP and test-and-hold programs, cannot ensure a safe, low-risk product for raw milk consumers.

In contrast, the 1999 Codex Alimentarius Commission (CAC) principles and guidelines document [6] that included one of the authors (MEC) specified that for objectivity, microbial risk assessments should be 'soundly based on science' and transparent. The recent evidence map analyses [1-3] document not only a coherent scientific basis for assessing benefits and risks simultaneously, but also feasible ecological and immunological mechanisms associated with protection from disease. Although the importance of physico-chemical factors (e.g., $\mathrm{pH}$ and temperature) and bioactive components including microbes naturally present in raw milks to supporting innate and acquired immunity and reducing pathogens are not new ideas for dairy food safety [7], the companion manuscripts increase transparency for the extensive mechanistic and clinical body of evidence on raw milk benefits and risks for both breastmilk [1,2] and cow milk [3]. Our companion manuscripts [1-3] emphasize that evidence for both benefits and risks must be weighed for 21st-century raw milks. Application of intentionally conservative assumptions for infectious disease risks based on outdated dogmas from 20th-century science is problematic in this decade of the 'microbiome revolution' that is transforming our paradigms for assessing and managing risks of infective and non-communicable diseases. Decision makers around the world may continue to erroneously assume that pasteurization is a 'silver bullet', a simple magical fix to complex issues around the choices for consuming milk. Particularly, such people may continue to believe pasteurization to significantly decrease risks and significantly increase benefits for donor breastmilk and ruminant milks despite extensive evidence to the contrary [1-3]. Clearly, 21st-century risk analysis methodologies can no longer ignore the natural milk microbiota and the changing paradigm of milk ecosystem interactions in health and disease.

In order to develop evidence-based decision support for regulating raw milks in the future, in our opinion, diverse stakeholders, including consumers, decision makers, scientific experts, and risk practitioners around the world, require opportunities for open dialogue as structured deliberations of available evidence for both breastmilk [2] and cow milk [3]. The workshops are envisioned to open the first cycles of analysis and deliberation for raw milks in this century based on analytic-deliberative process as framed by a National Academies committee [8] that included one of the authors (DWN). Consistent with technological advances in microbiology and dairy science, as well as the CAC principles and guidelines, the international workshops will address the 'state of the science' and uncertainties, the potential biases and outdated assumptions about risks, and the impacts of new data and alternative assumptions for those who choose raw donor breastmilk for their infants and raw cow's milk for children and adults. We will welcome participation of dairy and veterinary scientists and researchers as planning for international workshops continues.

References

1. Coleman ME, Dietert RR, North DW, Stephenson MM (2021) Enhancing human superorganism ecosystem resilience by holistically 'managing our microbes'. Applied Microbiology 1(3): 471-497.

2. Coleman ME, North DW, Dietert RR, Stephenson MM (2021) Examining evidence of benefits and risks for pasteurizing donor breastmilk. Applied Microbiology 1(3): 408-425.

3. Dietert RR, Coleman ME, North DW, Stephenson MM (2022) Nourishing the human holobiont to reduce the risk of 
non-communicable diseases: A cow's milk evidence map example. Applied Microbiology 2(1): 25-52.

4. Oikonomou G, Addis MF, Chassard C, Nader-Macias ME, Grant I, et al. (2020) Milk microbiota: what are we exactly talking about? Frontiers in Microbiology 11 60.

5. Food Standards Australia New Zealand (FSANZ) (2009) Microbiological risk assessment of raw cow milk.
6. Codex Alimentarius Commission (CAC) (1999) Principles and guidelines for the conduct of microbiological risk assessment.

7. Hull RR (2007) Challenging current concepts in dairy food safety. Australian Journal of Dairy Technology 62(2):100-102.

8. National Research Council (NRC) (1996) Understanding Risk: Informing Decisions in a Democratic Society. National Academy Press, Washington, DC, US. 